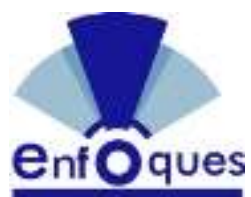

Enfoques. Revista de Investigación en Ciencias de la Administración http://doi.org/10.33996/revistaenfoques.v3i11.65 No. 11| Volumen 3 | Julio - Septiembre 2019 http://revistaenfoques.org ISSN: $2616-8219$ pp. $188-201$

\section{GESTIÓN DE INVENTARIO EN EMPRESAS DISTRIBUIDORAS DE MATERIA PRIMA DEL SECTOR PANADERO EN EL ESTADO ZULIA}

\author{
MANAGEMENT INVENTORY IN THE DELIVERY COMPANIES OF \\ THE RAW MATERIALS FOR THE BAKERY SECTOR IN \\ ZULIA STATE
}

Artículo recibido marzo 2019 | Arbitrado abril 2019 | Publicado 01 de julio 2019

\section{Resumen}

La presente investigación tuvo como objetivo describir los elementos que conforman la gestión de inventario en las empresas distribuidoras de materia prima del sector panadero en el estado Zulia. Por su alcance, se consideró una investigación descriptiva, con un diseño no experimental, de campo y de tipo transeccional. La población la conformaron nueve (9) sujetos entre gerentes y supervisores. Para la recolección de los datos se utilizó la técnica de la encuesta con un instrumento de frecuencia bajo la escala de Likert conformada por cinco (5) opciones de respuesta. La validez se realizó a través del juicio de expertos. La confiabilidad fue calculada por el coeficiente de Alfa de Cronbach y se ubicó en 0,962, lo cual indicó que el instrumento era altamente confiable. El análisis de los datos se realizó mediante la estadística descriptiva, con base en la media. Los resultados obtenidos muestran una mediana aplicación de la gestión de inventario en dichas empresas, lo que le permite cumplir con las necesidades de sus clientes.

Palabras clave: Gestión de inventario; sistema de control de inventario; costos de inventario; proceso de almacenamiento

\begin{abstract}
The purpose of this research was to describe the elements that make up inventory management in the raw material distribution companies of the bakery sector in Zulia state. Due to its scope, a descriptive investigation was considered, with a non-experimental, field and transectional design. The population was made up of nine (9) subjects between managers and supervisors. For the data collection, the survey technique was used with a frequency instrument under the Likert scale consisting of five (5) response options. The validity was made through expert judgment. Reliability was calculated by Cronbach's alpha coefficient and stood at 0.962, which indicated that the instrument was highly reliable. Data analysis was performed using descriptive statistics, based on the average. The results obtained show a medium application of inventory management in these companies, which allows it to meet the needs of its customers.
\end{abstract}

Key words: Inventory management, inventory control system, inventory costs, storage process
Damarick Diomara Pacheco B. damarickp@gmail.com

Impormaquinas \& Outsoursing S.A.S y Universidad del Zulia, Venezuela

Ingeniero Mecánico, egresado de la Universidad del Zulia. Magister Scientiarum en Gerencia de Operaciones. Universidad del Zulia. 


\section{INTRODUCCIÓN}

En la actualidad las organizaciones se encuentran envueltas bajo un entorno dinámico y variable que requieren de herramientas exitosas para afrontar los embates de la globalización. Esta situación genera cada vez mayor competitividad en las empresas, lo cual es necesario para garantizar la permanencia de las mismas en el mercado.

A escala mundial, según el Fondo Monetario Internacional (FMI, 2016), en los últimos años algunas economías se afianzaron, en tanto que otras, en particular las economías emergentes y en desarrollo, sufrieron aprietos debido al desplome de los precios de las materias primas, así como el endurecimiento de las condiciones financieras. Además, dicho organismo considera que el deterioro refleja consecuencias macroeconómicas esperadas de un sustancial aumento de la incertidumbre, inclusive en el ámbito político. Se proyecta que esta incertidumbre debilitará la confianza y la inversión.

No obstante, en un acelerado proceso de cambio, se está enfatizando la necesidad de reorganizar nuevas modalidades en las operaciones de las empresas, el nuevo escenario exige, tanto de los gobiernos como de los empresarios, la formulación de estrategias para adaptarlas exitosamente al mercado. Sin embargo, mientras la recuperación mundial continúa luchando por afianzarse, en América Latina y el Caribe se prevé que en 2016 el crecimiento sea negativo por segundo año consecutivo. Tal es el caso de América del Sur que se ha visto notablemente afectada por la caída de los precios de materias primas y del petróleo (FMI, 2016).

Al mismo tiempo, el incremento de la población, el crecimiento de la clase media en Latinoamérica y su capacidad adquisitiva, estimulan la demanda de bienes y servicios. Esta situación ha traído como consecuencia que los gerentes se sientan obligados a modificar las estrategias tradicionales para dar mayor relevancia a la planificación de las actividades como a la toma de decisiones, especialmente, en el manejo de los recursos. Además, una organización que aspire a sobrevivir en el contexto actual, tiene que ser capaz de interpretar las demandas que recibe para responder a ellas; el diálogo interno conllevará a que las organizaciones puedan alcanzar las metas, valores y misiones establecidas dentro de las mismas.

Por otra parte, en una organización, el rendimiento de un equipo de trabajo supone todo un reto, los propios trabajadores como clientes internos influyen en gran medida en la manera de alcanzar un objetivo; tanto la motivación como la estimulación de los equipos de trabajo son clave a la hora de desempeñar las tareas asignadas $y$ contribuyen en el proceso a conseguir un equilibrio entre la meta y lo que se tiene.

No obstante, uno de los recursos más grande e importante de la empresa, al igual que el capital humano, lo constituye el inventario o proceso logístico, por ello requiere de una adecuada administración ya que permite seguir la trazabilidad de un producto, mediante un conjunto de técnicas para acortar tiempos, reducir costes de almacén, transporte y conseguir que el producto llegue al destinatario final cuándo lo necesita.

Cabe destacar que la gestión de inventario permite afianzar cómo reorientar, en los casos necesarios, la posición competitiva de las empresas a través de información y recomendaciones para que introduzcan posibles mejoras en las 
estrategias de los procesos de inventario en el almacenamiento de materia prima.

En efecto, existen numerosas razones por las cuales los inventarios están presentes en un canal de suministros; aun así, en años recientes, el mantenimiento de inventarios ha sido totalmente criticado como innecesario y antieconómico. "Las razones para mantener los inventarios se relacionan con el servicio al cliente o para costear economías indirectamente derivadas de ellos; disponer de inventarios para los clientes no sólo puede mantener las ventas, sino que también puede aumentarlas" (Ballou, 2004, p. 328).

Por otra parte, en el manejo estratégico de una empresa, la gestión de inventarios es un factor determinante; los directores de operaciones establecen sistemas para gestionar el inventario, determinan cómo se pueden clasificar los artículos y cómo pueden mantener registros de inventarios exactos.

En relación a lo indicado anteriormente, la falta de automatización tecnológica relacionadas a la gestión de inventario se puede traducir como resultados no acertados, el rastreo manual quita mucho tiempo y no refleja buenos efectos que regularmente se convierten en pérdida de recursos o dinero. Es por esto, que es recomendable que los inventarios sean manejados mediante software; los problemas más comunes son debido a que no todos saben cómo utilizarlos; además, la falta de entrenamiento del personal puede afectar severamente la habilidad para estar al día con los pedidos por la carencia de criterio a la hora de clasificar productos.

Es importante destacar que, al no realizar adecuadamente las funciones de registros de mercancía no se podrá contar con información suficiente y útil para minimizar costos de producción, mantener un nivel de inventario óptimo. La falta de registros podría acarrear a una situación de exceso de inventario que puede deducirse en descontrol y disminución de la liquidez o insuficiencia de inventario donde se podrían perder ventas y clientes debido a que no se cuenta con la cantidad de productos necesarios para cumplir con los compromisos.

En el caso específico de las empresas distribuidoras de materia prima del sector panadero en el estado Zulia, las mismas se encuentran trabajando a media capacidad debido a un déficit de insumos que le impide satisfacer la demanda y garantizar el abastecimiento.

Cabe destacar, que la industria panificadora es uno de los brazos de la industria nacional, con productos de consumo masivo y representa el $40 \%$ de la distribución nacional, lo cual la convierte en un canal estratégico, ya que las panaderías están en todos los espacios de la geografía y en horarios extensos. En razón de esto, el continuo suministro de materias primas es vital para el abastecimiento nacional efectivo y congruente (Ramos, 2016).

Frente al escenario económico del país, las empresas distribuidoras de materia prima del sector panadero tienen como reto mantener inventarios de insumos, para asegurar la distribución oportuna y eficiente de productos, evitando que sus clientes paralicen sus actividades.

En este contexto, las empresas distribuidoras de materias primas del sector panadero, requieren de una gestión de inventario óptima para mantener niveles de existencias que les permita cumplir las peticiones de sus consumidores cuando estos así lo requieran. Además, se hace necesario implementar formas eficientes de 
administrar los departamentos de compra y almacén, ya que la falta de criterio al clasificar y ordenar productos puede afectar el flujo de trabajo al momento de cumplir con los compromisos. (Ramos, 2016).

En el mismo orden de ideas, en entrevistas no estructuradas realizadas al personal de las empresas del sector, indicaron que debido a la gran variedad de productos que manejan las empresas objeto de estudio, en algunos casos incurren en altos costos por fallas en la gestión de inventario, a consecuencia de situaciones tales como, manejo de inventario excesivo o por el contrario reducido, errores en las cantidades registradas por unidades de existencias, confusión en el despacho de productos similares, casos de caducidad y obsolescencia, deterioro de productos, mal manejo y ubicación de materiales, entre otras situaciones que generan inconvenientes al cierre de inventario en un periodo.

En la actualidad, estas empresas tienen la necesidad de mantener una adecuada rotación de su inventario, por lo que se hace imprescindible el uso de sistemas de control que les permita conocer el nivel de existencias real, las condiciones de sus productos y contar con un adecuado proceso de almacenamiento que les permita ofrecer calidad de servicio, en el menor tiempo requerido.

Ante la situación planteada, surge la interrogante de investigación: ¿Cómo son los procesos que conforman la gestión de inventario en las empresas distribuidoras de materia prima del sector panadero en el estado Zulia?

Son múltiples las razones que justifican la existencia y control de los inventarios, considerándose entre ellas que prevén la escasez, generan ganancias adicionales al momento de producirse alzas en los precios de los productos, garantizan la satisfacción del cliente al brindársele oportunamente el bien requerido, entre otras.

Según, la Facultad de estudios a distancia (s/f, p. 5) (Faedis) "un inventario es una provisión de materiales que tiene como escenario principal facilitar la continuidad del proceso productivo y la satisfacción de la demanda de los clientes".

De igual manera, Muller (2005, p. 1) sostiene que "los inventarios de una compañía están constituidos por sus materias primas, productos en proceso, los suministros que utiliza en sus operaciones y los productos terminados".

Por su parte, Vidal $(2010$, p. 23) sostiene que en la actualidad "los inventarios son vistos como un riesgo potencial mayor", esto en razón de situaciones generadoras de eventos como el riesgo de obsolescencia tecnológica, el cual se hace presente en productos que poseen ciclos de vida cortos, lo que exige que sean manejados con mayor cuidado. Ante esto, según dicho autor, la estrategia radica en estimar su nivel óptimo en función de cada caso en particular, tomando en consideración elementos como: sector productivo, características propias de la empresa, localización, estrategia competitiva y mercado, entre otros.

Así, el inventario, como elemento clave de toda organización debe ser administrado eficientemente, ya que según Ehrhardt y Brigham (2007) persigue como objetivos fundamentales garantizar la operatividad de la empresa con los productos en existencia y conservar niveles óptimos que permita minimizar los costos totales (de pedido y de mantenimiento). En razón de esto, se considera que una eficiente gestión de inventario permite a la organización mantener un nivel adecuado de existencias 
que asegure el abastecimiento en cualquier época, lo cual redunda en un servicio constante y eficiente al cliente.

En efecto, la gestión de inventarios es un punto determinante en el manejo estratégico de toda organización. Las tareas correspondientes a la gestión de un inventario se relacionan con la determinación de los métodos de registro, los puntos de rotación, las formas de clasificación y los modelos de reinventario, determinados por los métodos de control.

En este sentido, Lacalle (2013), define la gestión de inventario como el conjunto de actividades desarrolladas por la organización que permiten conocer con exactitud el volumen de ventas o salidas de existencias para así determinar la cantidad precisa que se deberá solicitar a los proveedores. Adicionalmente, menciona que la gestión de inventario conlleva a dos tipos de actividades: determinar en qué momento pedir y en qué cantidad hacerlo, y mantener actualizada la información de materiales disponibles.

En razón de lo anteriormente planteado puede inferirse que una buena gestión de inventario es fundamental en las empresas, ya que de ésta depende la continuidad del proceso de producción, por lo que Muller (2005), plantea que la gestión del inventario es una medida crítica utilizada por cada empresa. Si existe una deficiente gestión de inventario, se puede dar lugar a importantes pérdidas financieras.

A su vez, Vidal (2010, p. 23) considera que "ante la globalización actual de la economía, la administración de inventarios, la planeación de la producción, y la estrategia corporativa, están íntimamente ligadas". Esta sincronía o ajuste estratégico debe alcanzarse a través de la interrelación de las capacidades competitivas de la empresa y su correspondiente cadena de abastecimiento.

Así, la gestión de inventarios puede ser entendida como el proceso que monitorea la entrada y salida de inventario con la finalidad de mantener y controlar los niveles pertinentes, garantizando un servicio correcto y puntual a sus clientes, dentro del menor coste posible. Para efectos de esta investigación se consideró su estudio mediante las dimensiones: procesos, sistemas de control y costos operacionales.

Con relación a la definición de proceso, Ogalla (2005) lo define proceso como un conjunto de actividades que se realizan para satisfacer las necesidades y expectativas del cliente, lo cual también suele llamarse cadena de valor o proceso de negocio. Según este autor los procesos se clasifican en: Estratégico, de negocio y de soporte.

Por su parte, Barba (2006) menciona que el proceso es un conjunto de actividades interrelacionadas capaces de, a partir de uno o varios factores, producir como resultado un producto o servicio óptimo que represente un valor para el cliente.

En efecto, la adecuada integración de las actividades involucradas en la gestión de inventario, permiten de una u otra manera mantener la continuidad de abastecimiento o compra a fin de asegurar la continuidad de los procesos del negocio que realiza la organización.

Así, Escudero (2011) refiere que la gestión de compras o proceso de compras es el conjunto de operaciones que realiza la empresa para abastecerse de los materiales necesarios cuando tiene que realizar las actividades de fabricación o comercialización de sus productos. Para este autor el proceso de compra consta de las siguientes fases: Planificación de las compras, análisis de las necesidades, solicitud de ofertas y 
presupuestos, evaluación de las ofertas recibidas, selección del proveedor, negociación de las condiciones, solicitud del pedido, seguimiento del pedido y los acuerdos.

De igual manera, Heredia (2013), considera que el proceso de compras o adquisiciones, consiste en suministrar de manera ininterrumpida, materiales, bienes y/o servicios, para incluirlos de manera directa o indirecta a la cadena de producción. Estos bienes y/o servicios, deben proporcionarse en las necesidades adecuadas, en el momento solicitado, con el precio acordado y en el lugar requerido por el cliente, dentro de unos tiempos estipulados previamente.

En efecto, para conocer las necesidades de aprovisionamiento es necesario establecer una planificación del proceso de compra, para lo cual se analiza la demanda, es decir, tanto la cantidad que se tiene previsto vender como los niveles óptimos de inventario que la empresa debe tener en el almacén para satisfacer dicha demanda. Por lo tanto, la planificación de las compras persigue determinar la cantidad de producto que la empresa debe tener en un momento determinado. Entre los aspectos que engloba, se tiene: Selección de proveedores, evaluación de la mejor alternativa, proceso de compra y compra. (Ayala, 2016).

Con base a lo expuesto por los anteriores autores, se concluye que el proceso de compra es aquel encargado de conseguir los productos y bienes necesarios para el buen funcionamiento de la empresa, en las cantidades y fechas requeridas, de manera que ésta pueda concretar los objetivos marcados, utilizando las herramientas necesarias que permitan mantener un nivel de inventario óptimo al menor coste. El proceso de compra abarca varias fases antes de tomar la decisión de compra, que van desde el reconocimiento de la necesidad, planificación, búsqueda de información, evaluación de las alternativas y decisión de comprar. Una vez cubiertas estas fases se inicia el proceso de compra como tal que está compuesto por los siguientes elementos: planificación, selección de proveedor, negociación de la compra, solicitud de pedido y registro de compra.

Dentro de las actividades de gestión de inventario el proceso de almacenamiento es de vital importancia, ya que la manipulación y ubicación del material en el almacén puede evitar deterioros y diferencias de inventario, así como agilizar el abastecimiento a las líneas de producción para facilitar la preparación de los envíos a los clientes. Este proceso trata tanto de la recepción como de los movimientos de materias primas.

Según Rubio y Villarroel (2012), el almacenamiento es un proceso técnico del abastecimiento y sus actividades están referidas a ubicación, custodia y control de los bienes. Todo ello para asegurar sus condiciones óptimas, evitar una utilización no autorizada y facilitar su traslado a los usuarios que los requieren.

A su vez, Escudero (2011), indica que el proceso de almacenamiento está compuesto por el conjunto de actividades o tareas que tienen por objeto almacenar y custodiar aquellos inventarios que no están en proceso de transformación o se encuentran en trayecto desde los proveedores o hacia los clientes.

Asimismo, Ballou (2004), apunta que el sistema de almacenamiento puede separarse en dos funciones importantes: almacenamiento y el manejo de materiales. El manejo de materiales se refiere a las actividades de carga y descarga, al traslado del producto hacia y desde las diversas 
ubicaciones dentro del almacén a recoger el pedido. El almacenamiento simplemente es la acumulación de inventario en el tiempo. Se eligen diversas ubicaciones en el almacén y diferentes periodos de tiempo, dependiendo del propósito del almacén.

Así, el proceso de almacenamiento constituye una herramienta de vital importancia dentro de la gestión de inventario puesto que la calidad de los bienes o servicios ofrecidos por una organización dependen en gran medida del almacenamiento y manejo apropiado de estos. El almacenamiento permite mantener en forma organizada las mercancías que se requieran para el abastecimiento sistemático, con el fin de posibilitar la función de despacho.

El proceso de almacenamiento engloba los siguientes elementos: recepción, sistema de información, tecnología, almacenamiento, manejo de materiales y despacho.

\section{Sistema de control de inventario}

Otra de las dimensiones que conforman la variable en estudio es el sistema de control de inventarios, que según Chase y Jacobs (2014), un sistema de control de inventario es el conjunto de políticas y controles con los cuales se vigilan los niveles de inventario y determinan los que se van a mantener, el momento en que es necesario reabastecerlo y las dimensiones de los pedidos.

Según Muñiz (2013), el control de las existencias se relaciona con funciones que permiten obtener información sobre los inventarios y realizar la verificación de la misma, así como de su valoración; realizar el control de inventario (rotación de existencias, obsolescencia, diferencias de inventarios, baja de inventario) y obtener la correcta información para la contabilización sobre la variación de las existencias.

Heizer y Render (2008), indican que los directores de operaciones necesitan establecer sistemas para gestionar los inventarios. Entre los elementos a considerar para los sistemas de inventario están: cómo se pueden clasificar los artículos del inventario y cómo pueden mantener registros de inventarios exactos.

En efecto, los sistemas de control de inventarios permiten, por una parte, minimizar la inversión del inventario, puesto que los recursos que no se destinan a ese fin se puede invertir en otros proyectos, por otro lado, asegurarse de que la empresa cuente con inventario suficiente para hacer frente a la demanda cuando se presente, para que las operaciones de producción y venta funcionen sin obstáculos.

Ante lo planteado, es importante mencionar a Krajewski, Ritzman y Malhotra (2008), quienes expresan que para estudiar los inventarios hay que clasificarlos según la forma en la que se crearon. En este contexto, existen cuatro (4) tipos de inventarios: inventario de ciclo, de seguridad, de previsión y en tránsito. Para objeto de la investigación sólo se consideraron de seguridad, previsión y en tránsito.

Un sistema de inventarios como conjunto de normas, métodos $y$ procedimientos aplicado de manera sistemática busca planificar y controlar los materiales que se emplean en una organización. Este sistema puede ser manual - automatizado. Muchos autores los clasifican dependiendo del enfoque, para sustento de esta investigación se tomarán en cuenta los siguientes: $A B C$, máximos y mínimos, revisión continua, exactitud de registros, e inventarios obsoletos, entre otros. 
Con relación a la dimensión costos operacionales, esta ofrece información que los gerentes necesitan para tomar decisiones que deriven en un desempeño extraordinario. El estudio de los costos ayuda a comprender mejor la manera en que los gerentes y administradores contribuyen a las operaciones, los prepara para desempeñar también papeles de liderazgo. Muchas empresas utilizan la información de la contabilidad administrativa de los costos para elegir una estrategia, comunicarla, y determinar la mejor forma de ponerla en marcha.

Arredondo (2015), indica que los costos se deben considerar como elementos clave de la gerencia en todas las actividades de planeación, control y formulación de estrategias ya que proporcionan las herramientas contables indispensables para lograr el buen funcionamiento de algunas fases del proceso administrativo como son: la planeación, el control y la evaluación de las operaciones.

En la fase de planeación, la contabilidad de costos hace proyecciones a futuro a través de presupuestos, con esto se pueden determinar los costos en cuanto a materiales, sueldos, salarios y los gastos de fabricación que estén involucrados en la elaboración de un producto.

Cuando se habla de la fase de control, la contabilidad de costos hace referencia al tiempo presente, ya que compara los resultados reales que se obtienen con lo que se presupuesta en la fase de planeación, en esta fase se pueden identificar algunas fallas operativas que nos permiten llegar a la maximización de utilidades.

Por último, en la fase de evaluación se involucra un análisis crítico de los resultados que se obtuvieron realmente, se analizan los problemas que surgieron y las desviaciones que se obtuvieron con respecto a los resultados previstos, de tal forma que se puedan eliminar o aislar las causas que los originan.

Castrillón y Berrío (2008), definen el costo como el desembolso que reporta un beneficio futuro, por consiguiente, es capitalizable. Es la medida de valoración del consumo realizado o previsto por la aplicación racional de los factores para la obtención de un producto, trabajo o servicio. La rama de la contabilidad es un sistema de información que clasifica, acumula, controla y asigna los costos para determinar los costos de actividades, procesos y productos a fin de facilitar la toma de decisiones, la planeación, así como el control administrativo

\section{MÉTODO}

La presente investigación se ubicó en el estadio descriptivo, siendo la variable a estudiar gestión de inventario. El diseño de la investigación fue considerado no experimental, de campo y transeccional. Para la recolección de datos la técnica utilizada fue la encuesta y el instrumento un cuestionario conformado por 62 ítems con preguntas de alternativas de respuestas en un parámetro de frecuencia. La validez del instrumento se obtuvo a través del juicio de expertos. La confiabilidad fue calculada a través del coeficiente Alfa de Cronbach con un resultado de 0,96 lo que demuestra una alta confiabilidad del instrumento.

La unidad de estudio la conformaron las empresas distribuidoras de materia prima encargadas de distribuir y comercializar materia prima en el sector panadero en el estado Zulia registradas en el directorio de APPAEZ, en la modalidad de mayoristas, con servicio de venta y despacho de productos hasta la puerta de sus clientes, con procesos 
productivos similares entre ellas, con más de veinte años en el mercado. De esta manera se encuestaron los gerentes de las siguientes empresas: Dafilca de Occidente, C.A., Distribuciones Integrales, C.A., (Disinca), Distribuidora Beltrán, C.A. (Disbelca), Pandock del Lago, C.A. y Sumipan Occidente, C.A.

\section{RESULTADOS Y DISCUSIÓN}

El análisis de los datos se realizó mediante la estadística descriptiva y como medida de tendencia central la mediana. Para la interpretación de los datos se utilizó el baremo de interpretación que se presenta en la tabla 1.

Tabla 1. Baremo de interpretación

\begin{tabular}{ccc}
\hline Alternativas & Índice (\%) & Categoría \\
\hline Nunca & $0-19.9$ & Muy Baja Aplicación \\
Casi Nunca & $20-39.9$ & Baja Aplicación \\
Algunas Veces & $40-59.9$ & Mediana Aplicación \\
Casi Siempre & $60-79.9$ & Alta Aplicación \\
Siempre & $80-100$ & Muy alta aplicación \\
\hline
\end{tabular}

Con relación a la dimensión proceso, la cual obtuvo un índice porcentual promedio de 48 , tal como lo muestra la tabla 2 , que al ser comparado con el baremo de interpretación la ubica en la categoría mediana aplicación, lo cual indica que las empresas tienen un mediano desempeño en los procesos, específicamente los referidos a compras y almacenamiento que forman parte de su gestión de inventario. Este resultado puede considerarse como una consecuencia directa del comportamiento de sus indicadores cuyos resultados al ser comparados con el baremo de interpretación les ubicó en la categoría de mediana aplicación.

Tabla 2. Resultados de la dimensión proceso

\begin{tabular}{lcc}
\hline \multicolumn{1}{c}{ Indicadores } & Índice\% & Categoría \\
\hline Proceso de Compra & 48,4 & Mediana Aplicación \\
Proceso de Almacenamiento & 47,6 & Mediana Aplicación \\
\hline
\end{tabular}

Índice \% de la dimensión: 48 Mediana Aplicación

Estos resultados ponen de manifiesto la situación problema de las organizaciones en estudio y son opuestos a lo expresado por Moya (2007), cuando alude que el fin concreto de la gestión de compras consiste en cubrir las necesidades de la empresa con elementos exteriores a la misma, maximizando el valor del dinero invertido. Es así como una buena política de aprovisionamiento contribuye con los objeticos generales de la empresa a través de una buena gestión de inventario, en las mejores condiciones de abastecimiento y calidad. 
Asimismo, Ballou (2004) apunta que el proceso de almacenamiento constituye una herramienta de vital importancia dentro de la gestión de inventario puesto que la calidad de los bienes o servicios ofrecidos por una organización dependen en gran medida del almacenamiento y manejo apropiado de estos, ya que permiten mantener en forma organizada las mercancías que se requieran para el abastecimiento sistemático, con el fin de posibilitar la función de despacho.

Con relación a la dimensión sistema de control, la tabla 3 muestra como resultado un índice porcentual promedio de 57,8 que al ser comparado con el baremo de interpretación le ubica en la categoría de mediana aplicación, lo cual indica que las empresas en estudio tienen un mediano desempeño en la aplicación de sus sistemas de control después de cada transacción de entrada o salida de productos, además, medianamente cuentan con políticas de vigilancia que les permita mantener un inventario en estado actualizado.

Tabla 3. Resultados de la dimensión sistema de control

\begin{tabular}{ccc}
\multicolumn{1}{c}{ Indicadores } & Índice\% & Categoría \\
\hline Tipos de inventario & 73.2 & Alta aplicación \\
Tipos de sistemas de control & 42,5 & Mediana aplicación \\
\hline
\end{tabular}

Índice \% de la dimensión: 57,8 Mediana aplicación

Estos resultados se contraponen a los planteamientos de Chase y Jacobs (2014), quienes expresan que un sistema de control de inventario es el conjunto de políticas y controles con los cuales se vigilan los niveles de inventario y determinan lo que van a mantener; el momento en que es necesario reabastecerlo y las dimensiones de los pedidos. Por su parte Heizer y Render (2008), indican que la gerencia de operaciones es la encargada de establecer los sistemas para gestionar los inventarios. Entre los elementos a considerar para los sistemas de inventario están ¿cómo se pueden clasificar los artículos del inventario? ¿Cómo pueden mantener registros de inventarios exactos?
En cuanto a la dimensión costos operacionales, la tabla 4 muestra como resultado un índice porcentual promedio de 69,4 que al ser comparado con el baremo de interpretación le ubica en la categoría de alta aplicación. En lo que respecta a los indicadores que la componen se observa que el indicador denominado de pedido alcanzó resultados porcentuales de $81,9 \%$ que lo ubicaron en muy alta aplicación. En tanto que se observan oportunidades de mejoras para loa indicadores de mantenimiento, transporte y almacén, los cuales obtuvieron resultados $\quad(61,1 ; \quad 72,2 ;$ y $\quad 62,5$ respectivamente) que les ubicaron en la categoría de alta aplicación. 
Tabla 4. Resultados de la dimensión costos operacionales

\begin{tabular}{lcc}
\hline & \multicolumn{2}{c}{ Costos Operacionales } \\
\hline Indicador & Índice \% & Categoría \\
\hline De pedido & 81,9 & Muy alta aplicación \\
De mantenimiento & 61,1 & Alta aplicación \\
De transporte & 72,2 & Alta aplicación \\
De almacén & 62,5 & Alta aplicación \\
\hline
\end{tabular}

Con base en los resultados obtenidos se evidencia que las empresas evalúan los costos asociados a la gestión de inventario, consideran relevante el costo de pedido y de transporte para asignar el costo en el producto final. Con menor frecuencia se observa que las empresas asocian los costos de mantenimiento y almacén para asignarlos en los productos, ya que son tratados como gastos.

Sin embargo, a pesar de que la valoración de la dimensión costos operacionales se ubicó en la categoría de alta aplicación, es necesario examinar cuidadosamente los procedimientos de determinación de costos desde el punto de vista de su relevancia para la toma de decisiones involucradas con la gestión de inventarios. En síntesis, para una gestión de inventario eficaz es necesario realizar un análisis adecuado de los costos.

Los resultados anteriormente descritos están en concordancia con Arredondo (2015), quien indica que los costos se deben considerar como elementos clave de la gerencia en todas las actividades de planeación, control y formulación de estrategias, ya que proporciona las herramientas contables indispensables para lograr el buen funcionamiento de algunas fases del proceso administrativo como son: la planeación, el control y la evaluación de las operaciones.

Por su parte, Castrillón y Berrío (2008), definen el costo como el desembolso que reportará un beneficio futuro, por consiguiente, es capitalizable. Es la medida de valoración del consumo realizado o previsto por la aplicación racional de los factores para la obtención de un producto, trabajo o servicio.

Ante la postura de los autores anteriores, se puede inferir que las empresas distribuidoras de materia prima del sector panadero en el estado Zulia consideran sus costos asociados de pedido y transporte para determinar el costo de venta de un producto final, mientras que sólo un porcentaje de los de mantenimiento y almacén son asignados a los costos de los productos, en tanto que el otro porcentaje es tratado como gastos.

Finalmente, el análisis de la variable gestión de inventario arrojó un índice porcentual de 58,4, que al ser comparado con el baremo de interpretación le ubica en la categoría de mediana aplicación, tal como puede apreciarse en la tabla 5. 
Tabla 5. Variable gestión de inventario

\begin{tabular}{lll}
\hline Dimensión & Índice\% & Categoría \\
\hline Procesos & 48 & Mediana aplicación \\
Sistemas de Control & 57,8 & Mediana aplicación \\
Costos & 69.4 & Alta aplicación \\
\hline
\end{tabular}

Índice \% de la dimensión: 58,4 Mediana aplicación

En síntesis, las empresas distribuidoras de materia prima en el sector panadero llevan a cabo una mediana gestión de inventario, lo cual incide en el servicio brindado al cliente $\mathrm{y}$ en los niveles de inventarios adecuados para atender sus demandas. Además, el manejo de procesos y sistemas de controles que utilizan sólo les permite monitorear medianamente tanto las entradas como las salidas de productos, lo que impacta negativamente en la cuantificación de los productos en existencia. Igual situación se hace presente en cuanto a la información recabada, lo que influye en la toma de decisiones a la hora de fijar punto de pedido, las cantidades que se requieren y el momento en que lo requieren.

Con relación al proceso de compra y almacenamiento desarrollado por las empresas en estudio cuya categoría fue de mediana aplicación, se observan en ellos oportunidades de mejoras.

En efecto, las compras deben ser consideradas como una de las tareas más importantes dentro de la cadena de suministros, ya que una empresa puede mejorar considerablemente sus márgenes de beneficio siguiendo una política de aprovisionamiento adecuada. De igual manera, organizar y controlar las operaciones y flujos de mercancías del almacén de acuerdo con los procedimientos establecidos y normativa vigente, así como asegurar la calidad y optimización de la red de almacenes y/o cadena logística es básico para la mejora de la productividad de las empresas en estudio. Por lo tanto, el éxito de una buena gestión de inventario depende de forma directa de una buena gestión en los procesos de compras y almacenamiento.

Con respecto a los sistemas de control, también con resultados de mediana aplicación, lo cual indica que las empresas tienen una mediana aplicación de sus sistemas de control y que medianamente cuentan con políticas de vigilancia que les permite mantener un inventario en estado actualizado, después de cada transacción de entrada o salida de productos.

Por el contrario, los costos operacionales resultaron de alta aplicación, lo cual evidencia que las empresas evalúan los costos asociados a la gestión de inventario, consideran relevante el costo de pedido y de transporte para asignar el costo en el producto final. Con menor frecuencia se observa que las empresas asocian los costos de mantenimiento y almacén para asignar los costos en los productos, ya que son tratados como gastos.

Como resultado, la variable gestión de inventario de las empresas en estudio tiene un comportamiento en contradicción con lo expuesto por Muller (2005), quien plantea que la gestión del inventario es una medida crítica utilizada por cada empresa, pues una deficiente gestión de inventario puede dar lugar a importantes pérdidas financieras, en razón de esto se considera que la gestión de inventarios es un punto determinante en el 
manejo estratégico de toda organización. En razón de lo anteriormente planteado, puede inferirse que una buena gestión de inventario es fundamental en las empresas, ya que de ésta depende la continuidad del proceso de producción.

\section{CONCLUSIONES}

Para la dimensión procesos (compras), los resultados indican que posee una mediana aplicación, es decir, planifican medianamente sus necesidades para mantener un nivel de existencias por un periodo establecido, lo cual no les permite una adecuada especificación de la cantidad y fecha en que se deben generar los pedidos por lo que dependen del nivel de existencias que tengan a mano, lo que resta rentabilidad a la actividad de la empresa y genera insatisfacción de la demanda.

En cuanto al proceso de almacenamiento se evidenció que medianamente utilizan sistemas de información para tener control e integración de los procesos que abarcan todo tipo de gestiones de la empresa. Desde el inventario hasta la contabilidad requieren mejorar el desempeño de los métodos de manejo de materiales y tecnología de identificación de productos, ya que los avances tecnológicos de los últimos años permiten un desarrollo de las actividades de un modo más factible y rentable en este aspecto.

Con respecto a los sistemas de control de la gestión de inventario, se observó que cuando el riesgo de ruptura de inventario es muy alto estas empresas se manejan con existencias bajas y su aprovisionamiento lo realizan considerando el movimiento de productos durante ciertas temporadas. De igual manera su análisis puso de manifiesto mediana utilización de políticas de control, lo que les genera inconsistencias entre el físico y el sistema de información.

En cuanto a los costos de la gestión de inventario de las empresas en estudio, se evidenció que generalmente asumen los costos de las inversiones que realizan para transportar o manejar los productos hasta su almacén. En lo que respecta al costo de pedido y de transporte estos son considerados para asignarlos al costo en el producto final, en tanto que de los costos de mantenimiento y almacén sólo una parte del presupuesto se considera para asignarlos a los costos del producto, la otra parte es tratada como gastos.

En resumen, por ser la gestión de inventario la base de la actividad comercial, es una tarea compleja para estas empresas, que requiere un gran compromiso $y$ sensibilización por parte de los empresarios y sus empleados, ya que son múltiples las ventajas que pueden obtener principalmente al garantizar un servicio puntual a sus clientes, dentro del menor coste posible.

\section{REFERENCIAS}

Arredondo, M. (2015). Contabilidad y análisis de costos. Segunda edición. Grupo editorial

Ayala, J. (2016). Gestión de compras. Editorial Editex. España Comercial. Editorial McGraw-Hill. España. ISBN: 078-84-481-8543-5

Ballou, R. (2004). Logística. Administración de la cadena de suministros. Quinta Edición. Pearson Educación. México

Barba, E. (2006). Ingeniería Concurrente: Guía para su implantación en la empresa, Diagnóstico y Evaluación. Ediciones Gestión 2000, S.A. España. ISBN: 978-84-8088-611-6.

Castrillón, J. y Berrío, D. (2008). Costos para gerenciar organizaciones manufactureras, comerciales $y$ de servicios. 
Ediciones Uninorte. Colombia. ISBN: 978-958-8252-75-9

Chase, R. y Jacobs, R. (2014). Administración de las Operaciones. Producción y Cadena de Suministros. Décima tercera edición. McGraw-Hill Interamericana Editores, S.A. México

Ehrhardt, M. y Brigham, E. (2007). Finanzas corporativas. Segunda edición. México: Editorial Thomson

Escudero, M. (2013). Gestión logística y comercial. Ediciones Paraninfo, S.A. España

Escudero, M. (2011). Gestión de aprovisionamiento. Tercera edición. España. Ediciones Paraninfo, S.A.

Universidad Militar Nueva Granada. Facultad de Estudios a Distancia (s/f) (Faedis). Guía gestión de inventarios. Disponible en: http://www.umng.edu.co/facultadde-estudios-a-distancia.pdf. (Consulta: diciembre 15 de 2018)

Fondo Monetario Internacional (2016). Perspectivas económicas. Las Américas (informe WEO). Edición digital. Washington, D.C, USA. Publicación ISBN13: 978-1-47554-991-1

Heizer, J. y Render B (2008). Dirección de la producción y de operaciones. Decisiones tácticas. Octava edición. Pearson Educación. España.

Heredia, N. (2013). Gerencia de Compras. Segunda Edición. ECOE Ediciones. Colombia
Krajewski, L.; Ritzman, L.; Malhotra, M (2008). Administración de las operaciones. Procesos y cadena de valor. Octava edición. PERSON Educación. México

Lacalle, G. (2013). Gestión logística y comercial. Editex. España

Müller, M. (2005). Fundamentos de administración de inventarios. Traducción: Efraín Sánchez. Grupo Editorial Norma. Colombia

Muñiz, L. (2013). Cómo implantar y evaluar un sistema de gestión. Editorial Profit, $\mathrm{S}$. L. España

Ogalla, F. (2005). Sistema de gestión. Una guía práctica. Cómo pasar de la certificación de calidad, a un enfoque integral de gestión. Ediciones Díaz de Santos. España. ISBN: 978-84-7978-9534.

Ramos, T. (2016). Director de Fevipan (Federación Venezolana de Industriales de la Panificación y Afines). Expo Fevipan. Venezuela.

Rubio, J. y Villarroel, S. (2012). Gestión de pedidos y stock. Ediciones Aula Mentor. España. Publicación ISBN: 978-84-3695435-7. México

Vidal, C. (2010). Fundamentos de control y gestión de inventario. Programa de editorial Universidad del Valle. Cali. Colombia 